\title{
2-[18F]Fluoro-2-Deoxy-D-Glucose Positron Emission Tomography Definition of Lung Targets in Vero Stereotactic Body Radiation Therapy Workflow
}

Charles A Kunos* and John P Shanahan

Department of Radiation Oncology, Summa Cancer Institute, 161 North Forge Street, Akron, Ohio, USA

*Corresponding author: Charles Kunos, Department of Radiation Oncology, Summa Cancer Institute, G-90 161 North Forge Street , Akron, Ohio 44304, USA, Tel: 330-375-3557; Fax: 330-375-3072; E-mail: kunosc@summahealth.org

Received date: Apr 21, 2015, Accepted date: May 15, 2015, Publication date: May 19, 2015

Copyright: ( 2015 Kunos CA, et al. This is an open-access article distributed under the terms of the Creative Commons Attribution License, which permits unrestricted use, distribution, and reproduction in any medium, provided the original author and source are credited.

\begin{abstract}
This retrospective study explored 2-[18 F]fluoro-2-deoxy-D-glucose positron emission tomography (FDG-PET) as an aid in target delineation among a first-group of five patients with single or multiple moving lung tumors planned for Vero stereotactic body radiation therapy (SBRT). Computed tomography (CT)-based free-breathing, end-inspiration, and end-expiration lung target volumes generated a single internal target volume (ITV).

ITVs were compared to a thresholded $40 \%$ maximum standard uptake value FDG-PET target volume (CTVpet), a contour assumed to be a surrogate for lung target motion during quiet breathing. Planning target volumes (PTV) and relevant lung planning constraints determined clinical execution of Vero SBRT. A mean $28 \%$ PTV expansion by adding in a CTVpet contour to the CT-based ITV converted two (40\%) of five cases from SBRT fractionation (40 Gy in four every other day fractions) to conventional fractionation (30 Gy in ten daily fractions).

In all five cases, CTVpet contours captured target motion not enclosed by the CT-based ITV. Vero SBRT radiation plans achieved effective normal tissue sparing without compromise of PTV target coverage. A single instance of less than 30-day posttherapy grade 2 fatigue occurred; no pulmonary toxicity has been observed in the 3-month follow-up period. Thresholded CTVpet contours impacted target delineation and clinical delivery of Vero SBRT treatment.
\end{abstract}

Keywords: Vero; Radiosurgery; Stereotactic body radiation; PET; Lung cancer; Oligometastasis

\section{Introduction}

This study investigates the use of 2-[ $\left.{ }^{18} \mathrm{~F}\right]$ fluoro-2-deoxy-D-glucose positron emission tomography (FDG-PET) images for delineation of pulmonary tumor targets when treating sites of recurrent lung cancer or lung oligometastases by Vero stereotactic body radiation therapy (SBRT).

Because intracellular hexokinase traps ${ }^{18} \mathrm{~F}-\mathrm{FDG}$ as a sugar lookalike, PET has assisted in the spotting of and in the contouring of cancer targets for radiation treatment through image fusion, overlay, or side-by-side viewing [1-4]. As highly precise radiation delivery platforms become more mainstream, there has been a need for accurate anatomic and metabolic exactness of cancer targets [5].

SBRT has an established role in the treatment of primary lung cancer [6-9]. SBRT has an emerging role in palliative management of recurrent lung cancer or treatment of a limited number oligometastases to the lung [10-12].

The gimbaled Vero linear accelerator nested in a rotational gantry $\left( \pm 185^{\circ}\right)$ and pivoting O-ring $\left( \pm 60^{\circ}\right)$ represents a first-in-class radiation platform for delivery of ablative radiation dose with submillimeter accuracy [13-16].
Early clinical success for Vero treatment of lung tumors has been observed [16]. Bringing together metabolic FDG-PET images and respiration-phased computed tomography (CT) planning simulation images has not been entirely investigated for Vero SBRT treatment of lung tumors, as it has for other SBRT platforms [1].

This retrospective study discusses our initial experience with overlaid FDG-PET and CT images as a tool in moving lung target delineation for Vero SBRT radiation planning and treatment.

\section{Methods and Materials}

\section{Patient selection}

Summa Health institutional review board approval (Akron, Ohio, USA) was granted for this retrospective study. All patients provided written informed consent for intended palliative SBRT.

The first five Vero SBRT patients with moving lung targets had histologically-confirmed diagnoses of either recurrent lung cancer previously treated by chemotherapy $(n=2)$ or by radiochemotherapy $(\mathrm{n}=1)$, or a limited number ( $\leq 3$ targets) of pulmonary oligometastases $(n=2)$.

Patient demographics, tumor characteristics, and Vero SBRT treatment parameters are listed in Table 1. 


\begin{tabular}{|c|c|c|c|c|c|c|c|c|c|c|c|c|c|c|c|c|c|}
\hline \multirow[t]{2}{*}{ Pt. } & \multirow[t]{2}{*}{ Age } & \multirow[t]{2}{*}{ Sex } & \multirow[t]{2}{*}{ PS } & \multirow[t]{2}{*}{ No. } & \multirow[t]{2}{*}{ Indication } & \multirow[t]{2}{*}{ Fractionation } & \multicolumn{3}{|c|}{$\operatorname{GTV}\left(\mathrm{cm}^{3}\right)$} & \multirow{2}{*}{$\begin{array}{l}\begin{array}{l}\text { CTV } \\
\left(\mathbf{c m}^{3}\right)\end{array} \\
\text { CTVpet }\end{array}$} & \multicolumn{2}{|c|}{$\operatorname{ITV}\left(\mathrm{cm}^{3}\right)$} & \multicolumn{2}{|c|}{ PTV $\left(\mathrm{cm}^{3}\right)$} & \multicolumn{3}{|c|}{ Vero beam set-up } \\
\hline & & & & & & & GTVfb & GTVi & GTVe & & ITV & ITVpet & PTVitv & PTVpet & $\begin{array}{l}\text { No. } \\
\text { static }\end{array}$ & $\begin{array}{l}\text { No. } \\
\text { arc }\end{array}$ & 0 -ring $\left({ }^{\circ}\right)$ \\
\hline 1 & $63 \mathrm{y}$ & M & 0 & 1 & recurrent & 4 x $10 \mathrm{~Gy}$ & 4.7 & 4.8 & 5.1 & 4.5 & 10.1 & 10.8 & 35.4 & 38.8 & 0 & 2 & $-15,+10$ \\
\hline 2 & $76 \mathrm{y}$ & $\mathrm{F}$ & 0 & 3 & oligometastases & 4 x 10 Gy & 1.9 & 1.8 & 1.5 & 4.2 & 4.3 & 27.4 & 26.7 & 29.3 & 0 & 7 & $-15,+15$ \\
\hline 3 & $85 \mathrm{y}$ & M & 0 & 2 & oligometastases & 4 x $10 \mathrm{~Gy}$ & 8.5 & 8.7 & 6.4 & 50.8 & 22.9 & 59.3 & 78.2 & 146.0 & 6 & 1 & $-10,+10$ \\
\hline 4 & $58 \mathrm{y}$ & M & 2 & 1 & recurrent & 10 x 3 Gy & 110.6 & 121.5 & 130.0 & 107.5 & 156.8 & 175.4 & 246.6 & 265.7 & 8 & 1 & 0 \\
\hline 5 & $65 y$ & M & 0 & 1 & recurrent & 10 x 3 Gy & 136.0 & 136.4 & 128.2 & 131.0 & 188.7 & 204.2 & 319.4 & 339.6 & 8 & 0 & 0 \\
\hline
\end{tabular}

*Abbreviations: Pt.: Patient; No.: Number; y: Year; M: Male; F: female; PS: ECOG Performance Status; GTV: Gross Tumor Volume; fb: Free-Breathing; i: EndInspiration; e: End-expiration; CTV: Clinical Tumor Volume; PET: Positron Emission Tomography; ITV: Internal Target Volume; PTV: Planning Tumor Volume

Table 1: Overview of patient data related to lung target number and fractionation schedule.

\section{Vero SBRT planning process and treatment}

The current respiratory-motion management solution at Summa Health required one, or up to three, radio-opaque gold-coated fiducial markers to be implanted within four-centimeters of an intended SBRT target. Markers were positioned either by a CT-guided interventional radiology approach [17] or an electromagnetic navigation bronchoscopy approach [18]. To provide intrafraction and interfraction motion tracking, at least one marker was implanted as close as safely possible to the SBRT target center. Patients breathed freely-meaning no coaching or physical restraints were applied during marker placement, pretherapy imaging or CT simulation, or treatment.

Four to seven days after marker implantation, patients rested comfortably for CT simulation in a head-first treatment position with their arms above their head supported by an evacuated vacuum-bag immobilizer. Non-contrasted helical CT images (1-millimeter slice thickness, $120 \mathrm{kVp}, 350 \mathrm{mAs}$ ) spanned the entire thorax through the mid-abdomen.

A 64-slice Toshiba Aquilon LB scanner (Toshiba Medical Systems, Tustin, California, USA) equipped with a flat tabletop and two-pin knee sponge tabletop registry was used. Multi-phase CT image data sets included a free-breathing scan, a moderate inspiration breath-hold scan, and a moderate expiration breath-hold scan. The scanner did not have respiratory phase binning capability engaged.

FDG-PET images of the patient torso were acquired during quiet breathing from the orbitomeatal line to the upper thighs in a head-first supine treatment position (Siemens Biograph 6.0, Siemens Healthcare, Erlangen, Germany). FDG-PET and CT-simulation patient positioning might have been different. Emission images were obtained per institutional protocol after intravenous administration of a median 16 millicuries ( $\mathrm{mCi}$, range $15-19 \mathrm{mCi}$ ) of ${ }^{18} \mathrm{~F}-\mathrm{FDG}$. A certified medical physicist imported, overlaid, and co-registered single-phase FDG-PET images manually in the Vero iPlan treatment planning system (Brainlab, Inc., Munich, Germany). Rigid FDG-PET image propagation was done using benchmarked anatomic features. Fiducial markers were not used necessarily in image registration.

The treating physician contoured targets and non-targets on the referent free-breathing CT simulation scan set on the preset lung level/ window. The anatomic gross tumor volume (GTV) was drawn on each of the free-breathing (GTVfb), inspiration (GTVi), and expiration (GTVe) CT simulation scans. Using already-studied thresholded values for the FDG-PET scan $[1,4]$, a 40 percent maximum standard uptake value contour was drawn by the treating physician on the FDG PET images. This contour represented a metabolic FDG PET clinical target volume (CTVpet).

Radiation planning involved four Vero iPlan-computed target volumes. The first internal target volume (ITV) represented the union of GTVfb, GTVi, and GTVe volumes. A second ITVpet included the union of the ITV plus the CTVpet volume. Corresponding planning tumor volumes (PTV) were created by an isotropic 5-millimeter margin expansion of the ITV (i.e., PTVitv) or the ITVpet (i.e., PTVpet).

The treating physician or certified medical dosimetrist contoured nearby normal tissue organs such as the uninvolved lung, heart, esophagus, spinal cord, liver, and bilateral kidneys. Volumes $\left(\mathrm{cm}^{3}\right)$ were computed in iPlan and then compared by paired t-test statistics (SPSS 18.0, SPSS Inc., Chicago, Illinois, USA).

Treatments were delivered on a Vero SBRT treatment platform (Brainlab, Inc.; Mitsubishi Heavy Industries Ltd., Tokyo, Japan). Evenly spread $6 \mathrm{MV}$ static fields and/or $6 \mathrm{MV}$ dynamic arcs were used in planning (Table 1). Non-coplanar Vero O-ring orientations of up to $\pm 15^{\circ}$ were set manually for adjacent beams (Table 1 ). The multileaf collimator aperture was set to shape beams most often around a threemillimeter PTV contour expansion. Beam aperture optimizations if any and subsequent Monte Carlo dose calculations were done with a $2 \%$ variance level. Planning was completed on the referent freebreathing CT scan.

To determine the impact of the CTVpet volume on respiratory motion management, identical static and arc beam arrangements were applied to the PTVitv and PTVpet. Gimbal rotation was not used. An internal protocol for recurrent disease or oligometastases in the lung intended a palliative prescription of $40 \mathrm{~Gy}$ in four 10 Gy every other day fractions.

SBRT normal tissue planning constraints followed clinical protocol guidelines [19] and are found in Table 2. If Vero SBRT parameters could not be met, an alternative conventional fractionation prescription (30 Gy in ten 3 Gy daily fractions) was used. 
Citation: Kunos CA, Shanahan JP (2015) 2-[18F]Fluoro-2-Deoxy-D-Glucose Positron Emission Tomography Definition of Lung Targets in Vero Stereotactic Body Radiation Therapy Workflow. J Nucl Med Radiat Ther 6: 227. doi:10.4172/2155-9619.1000227

Page 3 of 5

\begin{tabular}{|l|l|l|l|}
\hline Structure & Metric & Volume & Accepted variation \\
\hline PTV & V40Gy & $\geq 95 \%$ & $\geq 90 \%$ \\
\hline minimum dose & $0.03 \mathrm{~cm}^{3}$ & $\geq 46 \mathrm{~Gy}(92 \%)$ & $\geq 45 \mathrm{~Gy}(90 \%)$ \\
\hline maximum dose & $0.03 \mathrm{~cm}^{3}$ & $\leq 60 \mathrm{~Gy}(120 \%)$ & $\leq 62.5 \mathrm{~Gy}(125 \%)$ \\
\hline Lung (minus GTV) & V20Gy & $\leq 10 \%$ & $\leq 15 \%$ \\
\hline & Mean dose & $\leq 8 \mathrm{~Gy}$ & $\leq 10 \mathrm{~Gy}$ \\
\hline Spinal cord & $0.03 \mathrm{~cm}^{3}$ & $\leq 15 \mathrm{~Gy}$ & $\leq 22 \mathrm{~Gy}$ \\
\hline Heart / Pericardium & $15 \mathrm{~cm}^{3}$ & $\leq 32 \mathrm{~Gy}$ & $\leq 36 \mathrm{~Gy}$ \\
\hline Esophagus & $0.03 \mathrm{~cm}^{3}$ & $\leq 27 \mathrm{~Gy}$ & $\leq 30 \mathrm{~Gy}$ \\
\hline & Mean dose & $\leq 18 \mathrm{~Gy}$ & $\leq 20 \mathrm{~Gy}$ \\
\hline $\begin{array}{l}\text { Brachial } \\
\text { nerve plexus }\end{array}$ & $0.03 \mathrm{~cm}^{3}$ & $\leq 24 \mathrm{~Gy}$ & $\leq 30 \mathrm{~Gy}$ \\
\hline Abbrevilin: PTV: Plang & & \\
\hline
\end{tabular}

*Abbreviations: PTV: Planning Tumor Volume; GTV: Gross Tumor Volume

Table 2: Vero stereotactic body radiation therapy lung treatment planning constraints.

Treatment workflow included patient-specific treatment plan verification on the Vero SBRT platform a few days prior to treatment initiation. On days of radiation delivery, anterior and lateral tattooed CT simulation laser triangulation marks and an infrared-tracked reference array were used for patient localization prior to radiation beam-on. Repeated stereoscopic x-rays confirmed internal target position and motion during quiet breathing. This involved the treating physician and radiation therapist verifying target localization prior to radiation beam-on for any individual static beam or dynamic arc. During tracked radiation delivery, an always-active cine electronic portal imaging device (EPID) provided 'real-time' verification of target motion within the multileaf collimator aperture. Vero SBRT treatment workflow demanded treating physician presence to monitor EPID output and the patient.

\section{Patient assessment and follow-up}

Patients were seen before course initiation, after each day's treatment, and four weeks following treatment for assessment of graded adverse events (Common Terminology Criteria for Adverse Events, version 4.0). Subsequent follow-up and restaging CT or FDGPET imaging were not uniform and done at the patient's oncology team's discretion afterward. As such, a formal SBRT target response evaluation cannot be done.

\section{Results}

\section{Patients}

Five patients had eight lung tumors of recurrent lung (3) or oligometastatic (5) origin identified on CT and FDG-PET images (Table 1). Median posttherapy patient follow-up was three months (25-75\% quartile: 3-5 months). All five patients had Vero-targeted disease controlled at three months postherapy. Two months after treatment, one patient died from non-target elsewhere disease progression.

\section{PTV volume expansion}

The average PTVpet volume was $28 \%$ larger (range: 6-87\%) relative to the PTVitv volume $(\mathrm{p}=0.13)$. Table 1 contains volume data for the GTVfb, GTVi, GTVe, CTVpet, ITV, ITVpet, PTVitv and PTVpet for all five patients individually. The CTVpet volume underestimated target respiratory motion in four $(80 \%)$ of five patients, as evident from comparison to its corresponding CT-based ITV. CTVpet contours qualitatively described target motion not captured in the matching CT-based ITV in three $(60 \%)$ of five patients (Figure 1$)$. The clinical impact of an enlarged PTVpet was the conversion of two (40\%) Vero SBRT fractionation plans to their corresponding conventional fractionation plans. The number of recurrent or oligometastatic targets (range: 1 to 3 ) did not influence clinical execution of Vero SBRT treatment.

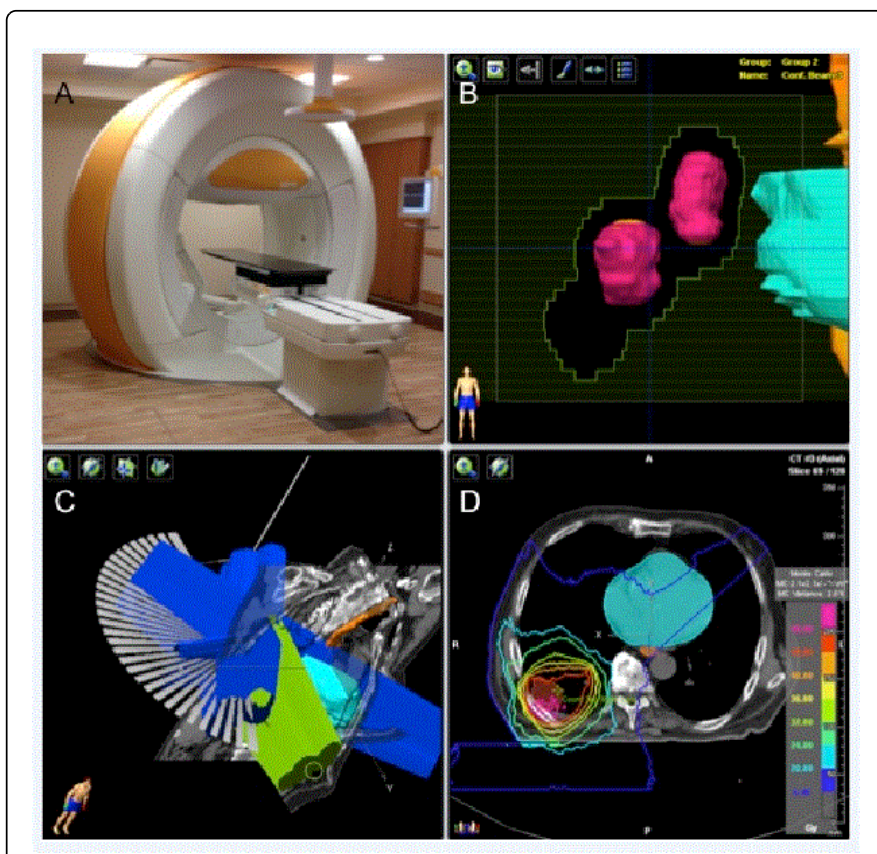

Figure 1: (A) The Vero SBRT platform. (B) Right anterior beameye-view of right lower lobe lung SBRT. CTVpet targets (magenta) are shown. Multileaf collimation (green outline) accounts for target motion during quiet breathing. Normal tissue contours for the heart (light blue) and esophagus (orange) are depicted to aid beam orientation selection. (C) Axial, coronal, and sagittal projections of single dynamic arc $(\tan )$ and six static treatment beams (green=active beam for $[\mathrm{B}]$ and blue=other treatment beams in radiation plan). (D) Axial projection of radiation dosimetry by Monte Carlo calculation. The prescription $40 \mathrm{~Gy}$ isodose contour is shown in gold, with accompanying isodose contours as labeled.

\section{Vero SBRT workflow and adverse events}

Vero treatment involved both static fields and dynamic arcs delivered over a total of 32 fractions (Table 1 and Figure 1). PTVpet coverage was $95 \%$ or greater in all five patients. SBRT normal tissue planning constraints (Table 2) were met in three $(60 \%)$ of five patients (6 [75\%] of 8 tumors). For the three Vero SBRT-treated patients, the mean lung V5\%, V20\%, and mean lung dose were 30\% (range: 25-35\%), 7\% (range: 2-11\%), and 2.1 Gy (range: 1.9 Gy-2.4 Gy), 
respectively. The two patients treated by conventional fractionation had a lung V5\% of $23 \%$ and $37 \%$, a lung V20\% of $5 \%$ and $7 \%$, and a mean lung dose of $1.7 \mathrm{~Gy}$ and $2.9 \mathrm{~Gy}$. The SBRT fractionated plans for these two conventionally treated patients had a lung V5\% of $44 \%$ and $36 \%$, a lung V $20 \%$ of $11 \%$ and $12 \%$, and a mean lung dose of 3.9 Gy and $2.4 \mathrm{~Gy}$, respectively.

In the 30-day period posttherapy, three (60\%) patients self-reported grade 1 fatigue relieved by rest. One (20\%) patient reported grade 2 fatigue limiting instrumental activities of daily living. No patient reported new cough, dyspnea, pneumonitis, or other pulmonary toxicity. No patient self-reported lingering fatigue or pulmonary toxicity at three-months posttherapy.

\section{Discussion}

This study evaluated FDG-PET as a means of precise target determination in patients with single or multiple moving lung tumors at the outset of our Vero SBRT program. Thresholded FDG-PET contours generated a non-significant PTVpet volume expansion $(\mathrm{p}=0.25)$ in the studied patients, with an average volume increase of 28\% compared to PTVitv. Importantly, PTVpet volumes impacted non-execution of Vero SBRT treatment when two plans exceeded the clinical risk level for pulmonary toxicity.

During the Vero SBRT planning process, the goal was to use thresholded FDG-PET metabolic data as a surrogate for inspirationexpiration lung target hysteresis. This notion was not new [1-3]. A prior observational study conducted in 27 patients suggested that a single $40 \%$ maximum standard uptake value benchmark sufficiently discriminated abdominopelvic SBRT clinical target volumes [1]. This prior observational study was supported in concept by non-small cell lung cancer data where best-matched FDG-PET thresholds were 15\% for tumors greater than five-centimeters, $24 \%$ for tumors three- to five-centimeters, and $42 \%$ for tumors less than three-centimeters [4]. Given the submillimeter precision of the Vero SBRT platform [13], the investigators sought as much pretherapy data on target motion for SBRT radiation planning.

In this study, six (75\%) of eight lesions had portions of their FDGPET signal outside their corresponding individual CT-based ITV. A reason for FDG-PET signal outside the ITV might be inadequate capture of tumor target motion by the used three-phase static-image CT simulation protocol in this study. Other investigators have used 10-phase static-image CT simulations and planning done on one single reference 4DCT end-of-exhale phase scan [16], which might better capture overall target motion. Surprisingly, FDG-PET-based contours drawn for these eight lesions underestimated CT-based ITVs in the majority (80\%). The assumption that a single $40 \%$ maximum standard uptake value benchmark be applied in Vero SBRT for lung tumors might not be valid due to fluctuating FDG uptake related to the breathing cycle [4]. It was reassuring that motion for all eight targets resided entirely within PTVpet-generated radiation field edges during 'real-time' EPID image tracking. Certainly, further research must be done before drawing broad conclusions on the utility of FDGPET for target discrimination in Vero SBRT planning.

Vero SBRT treatment was safe, with the caveat that the number of patients studied was small and clinical follow-up was short. The selfreported incidence of pulmonary or chest-organ toxicity was very low in the 30-day period posttherapy. Reversible grade 2 or higher fatigue symptoms (20\%) were in line with other SBRT trials (16\%, [3]). Fatigue in the SBRT setting was attributed both to intense volume- directed radiation and to radiation-induced nuclear and mitochondrial DNA damage demanding excessive deoxynucleotide triphosphates, as considered before [20]. But, other factors might be in play.

This exploratory study was not designed to have sufficient power to detect strict tumor volume constraints, differential FDG-PET signal intensity in the center versus periphery of lung tumors, or demonstrable errors in rigid versus deformable PET-to-CT registration. Small sample size, user-defined FDG-PET threshold settings, bias in assignment of toxicity criteria, and short clinical follow-up remained limits.

Indications for stereotactic body radiation therapy in the management of recurrent lung cancers and oligometastases continue to evolve. Radiotracers such as ${ }^{18}$ F-FDG will be used undoubtedly to identify and to define lung tumor targets being treated by stereotactic body radiation therapy. Future research directions in the investigator group question whether FDG-PET data contributes to target delineation under other treatment conditions, such as Vero SBRT dynamic tracking [16].

\section{Conflict of Interest}

The authors declare that the research was conducted in the absence of any commercial or financial relationships that could be construed as a potential conflict of interest.

\section{References}

1. Kunos CA, Debernardo R, Fabien J, Dobbins DC, Zhang Y, et al. (2011) 18FDG-PET/CT Definition of Clinical Target Volume for Robotic Stereotactic Body Radiosurgery Treatment of Metastatic Gynecologic Malignancies. J Nucl Med Radiat Ther Suppl 4.

2. Kunos C, Radivoyevitch T, Abdul-Karim FW, Faulhaber P (2011) 18Ffluoro-2-deoxy-D-glucose positron emission tomography standard uptake value ratio as an indicator of cervical cancer chemoradiation therapeutic response. Int J Gynecol Cancer 21: 1117-1123.

3. Kunos CA, Brindle J, Waggoner S, Zanotti K, Resnick K, et al. (2012) Phase II Clinical Trial of Robotic Stereotactic Body Radiosurgery for Metastatic Gynecologic Malignancies. Front Oncol 2: 181.

4. Biehl KJ, Kong FM, Dehdashti F, Jin JY, Mutic S, et al. (2006) 18F-FDG PET definition of gross tumor volume for radiotherapy of non-small cell lung cancer: is a single standardized uptake value threshold approach appropriate? J Nucl Med 47: 1808-1812.

5. Kunos C (2013) Image-guided motion management. OMICS J Radiology 2:e120.

6. Bral S, Gevaert T, Linthout N, Versmessen H, Collen C, et al. (2011) Prospective, risk-adapted strategy of stereotactic body radiotherapy for early-stage non-small-cell lung cancer: results of a Phase II trial. Int J Radiat Oncol Biol Phys 80: 1343-1349.

7. Chang JY, Balter PA, Dong L, Yang Q, Liao Z, et al. (2008) Stereotactic body radiation therapy in centrally and superiorly located stage I or isolated recurrent non-small-cell lung cancer. Int J Radiat Oncol Biol Phys 72: 967-971.

8. Fakiris AJ, McGarry RC, Yiannoutsos CT, Papiez L, Williams M, et al. (2009) Stereotactic body radiation therapy for early-stage non-small-cell lung carcinoma: four-year results of a prospective phase II study. Int J Radiat Oncol Biol Phys 75: 677-682.

9. Ricardi U, Filippi AR, Guarneri A, Ragona R, Mantovani C, et al. (2012) Stereotactic body radiation therapy for lung metastases. Lung Cancer 75: 77-81.

10. Trovo M, Minatel E, Durofil E, Polesel J, Avanzo M, et al. (2014) Stereotactic body radiation therapy for re-irradiation of persistent or 
Citation: Kunos CA, Shanahan JP (2015) 2-[18F]Fluoro-2-Deoxy-D-Glucose Positron Emission Tomography Definition of Lung Targets in Vero Stereotactic Body Radiation Therapy Workflow. J Nucl Med Radiat Ther 6: 227. doi:10.4172/2155-9619.1000227

Page 5 of 5

recurrent non-small cell lung cancer. Int J Radiat Oncol Biol Phys 88: 1114-1119.

11. Rusthoven KE, Kavanagh BD, Burri SH, Chen C, Cardenes H, et al. (2009) Multi-institutional phase $\mathrm{i} /$ ii trial of stereotactic body radiation therapy for lung metastases. J Clin Oncol 27: 1579-1584.

12. Widder J, Klinkenberg TJ, Ubbels JF, Wiegman EM, Groen HJ, et al. (2013) Pulmonary oligometastases: metastasectomy or stereotactic ablative radiotherapy? Radiother Oncol 107: 409-413.

13. Depuydt T, Penne R, Verellen D, Hrbacek J, Lang S, et al. (2012) Computer-aided analysis of star shot films for high-accuracy radiation therapy treatment units. Phys Med Biol 57: 2997-3011.

14. Depuydt T, Poels K, Verellen D, Engels B, Collen C, et al. (2013) Initial assessment of tumor tracking with a gimbaled linac system in clinical circumstances: A patient simulation study. Radiother Oncol 106: 236-240.

15. Poels K, Depuydt T, Verellen D, Engels B, Collen C, et al. (2013) A complementary dual-modality verification for tumor tracking on a gimbaled linac system. Radiother Oncol 109: 469-474.
16. Depuydt T, Poels K, Verellen D, Engels B, Collen C, et al. (2014) Treating patients with real-time tumor tracking using the vero gimbaled linac system: Implementation and first review. Radiother Oncol 112: 343-351.

17. Mendiratta-Lala M, Sheiman R, Brook OR, Gourtsoyianni S, Mahadevan A, et al. (2014) CT-guided core biopsy and percutaneous fiducial seed placement in the lung: can these procedures be combined without an increase in complication rate or decrease in technical success? Eur J Radiol 83: 720-725.

18. Harley DP, Krimsky WS, Sarkar S, Highfield D, Aygun C, et al. (2010) Fiducial marker placement using endobronchial ultrasound and navigational bronchoscopy for stereotactic radiosurgery: An alternative strategy. The Annals of thoracic surgery 89: 368-373.

19. http://www.rtog.org/clinicaltrials.

20. Kunos C, DeBernardo R, Radivoyevitch T, Fabien J, Dobbins D, et al. (2012) Hematological toxicity after robotic stereotactic body radiosurgery for treatment of metastatic gynecologic malignancies. Int J Radiat Oncol Biol Phys 84: e35-e41. 